\title{
Surgical Implications of Open Carpal Tunnel Surgery Considering Anatomical Variants of the Median Nerve Through the Transverse Carpal Ligament
}

\author{
William J Cobb* \\ Department of Human Anatomy, University of Medicine and Health Sciences, St Kitts West Indies
}

Submission: October 06, 2016; Published: November 22, 2016

*Corresponding author: William J Cobb, Department of Human Anatomy, University of Medicine and Health Sciences, St Kitts West Indies, Tel: 07452981539; Email: willcobb26@hotmail.co.uk

\begin{abstract}
Open Carpal tunnel surgery is a relatively common surgical procedure in the UK. Surgery is the most preferential option when symptoms have continued for six months or more and other less invasive treatments have been tried, and have failed. The success rate of carpal tunnel surgery in the UK has been surveyed, and according to research by East Kent NHS Hospitals, which looked at over 6,263 carpal tunnel operations, overall figures suggest positives for surgical intervention. Findings from East Kent NHS trust showed that out of the carpal tunnel operations performed [1]:

i. $49 \%$ had a total success rate with no further interventions being necessary;

ii. $7 \%$ showed symptoms becoming worse post -surgery;

iii. $6 \%$ went unchanged with symptoms neither improving nor deteriorating;

iv. $10 \%$ showed slight improvement in function; and

v. $28 \%$ showed marked improvement to function.

In order for surgery to have the most positive outcomes for the patient, it is empirical that surgical practitioners should have a detailed knowledge and understanding of the anatomy of the hand, as well as variant forms, before approaching a surgical procedure [2].
\end{abstract}

\section{Introduction}

Understanding of the underlying anatomy of the hand and possible variants of the median nerve through the transverse carpal ligament allows the surgical practitioner to develop safety measures when performing surgery so as to minimize the risk of nerve damage. The focus of this paper is on the median nerve and its varied course through the transverse carpal ligament. This paper will be looking at literature on the variant forms of the median nerve as previously identified in cadaveric studies and surgical analysis. It will also consider how variants may cause complications in open carpal tunnel surgery. The Causes of carpal tunnel syndrome have been sourced to a variety of things, such as repetitive strain injury, diabetes and rheumatoid arthritis, as well as various other pathologies [3].
The anatomical construction of the carpal tunnel itself presents like a closed compartment, regulating its own tissue fluid level for the purpose of flexor tendon movement. The roof of the carpal tunnel is the flexor reticulum or transverse carpal ligament, this acts as a flexor pulley for the flexor tendons [4]. The flexor reticulum attaches at the pisiform; hamate; scaphoid; and trapezium bones in the hand, thus forming the structure of the carpal tunnel see (Figure 1) below: [5]. The median nerve, as well as nine tendons, passes through the carpal tunnel these are arranged as follows: four flexor digitorum superficialis and four flexor digitorum profundus, as well as flexor pollicis longus [6].

The median nerve itself originates at the level of C5-T1 in the spinal column and has both sensory and motor control [7]. The sensory distribution of the median nerve is part of the thenar 
eminence and the three and a half digits radially [8] (Figure 1). The motor supply distribution of the median nerve is to the radial two lumbricals and the muscles of the thenar eminence. The median nerve is situated between the flexor reticulum and the tendons of the middle finger, flexor digitorum superficialis [9]. The tight anatomical spacing of the median nerve in relation to other structures within the carpal tunnel suggests that even a slight variance in tunnel diameter will cause the pulley system to vary functionally or fail

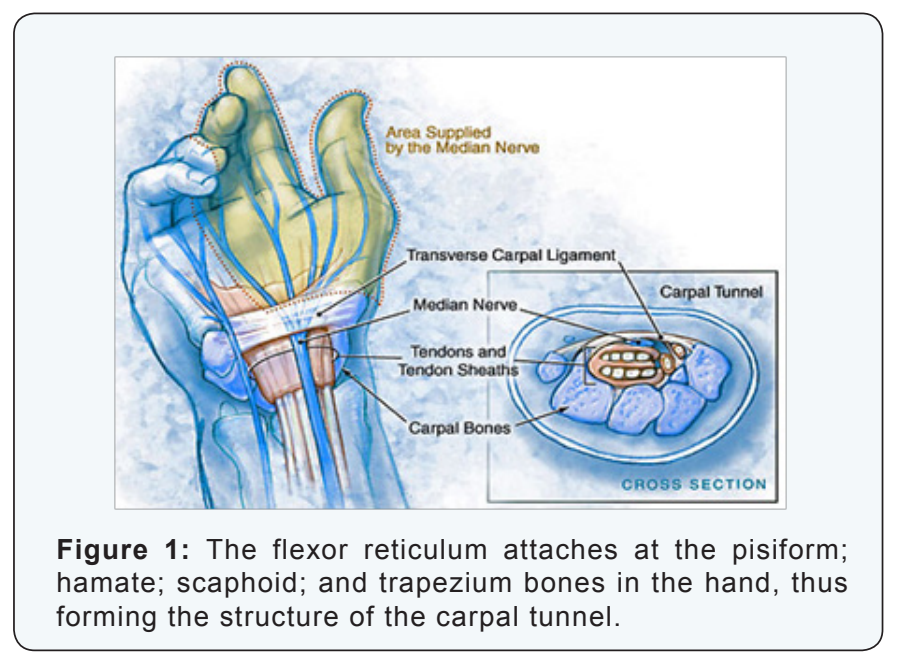

[10]. Was the first anatomical researcher to use groups to help describe the variations of the direction of the median nerve and tributaries through the carpal tunnel?

Poisel conducted acadaveric research project looking at the carpel tunnel of 100 cadavers, and concluded from this research that three distinct classification groups could be identified in relation to travel of the median nerve through the transverse carpal ligament. The groups created by Poisel enabled variations to be identified in a more ordered manner: (Group I) extraligamantous; (Group II) subligamentous; and (Group III) trans-ligamentous. Poisel found that the extraligamantous variant could be identified in 46 specimens. In 31 specimens the subligamentous variant was seen, and in 23 specimens the transligamentous variant was seen. The extraligamantous thenar branch was described by Poisel as being the most common (or standard anatomy as seen in the majority of the population sample).

Lanz [11] went about updating Poisel's grouping system to include more variant forms of the travel of the median nerve by undertaking a study looking at the course of the median nerve through the carpal tunnel in 246 hands during surgical procedures. Lanz identified 29 variations in the path of the median nerve through the carpal tunnel.

i. 18 hands showed accessory branches at the distal portion of the tunnel;

ii. 7 hands showed a high division of the median nerve; and iii. 4 hands showed accessory branches proximal to the tunnel.

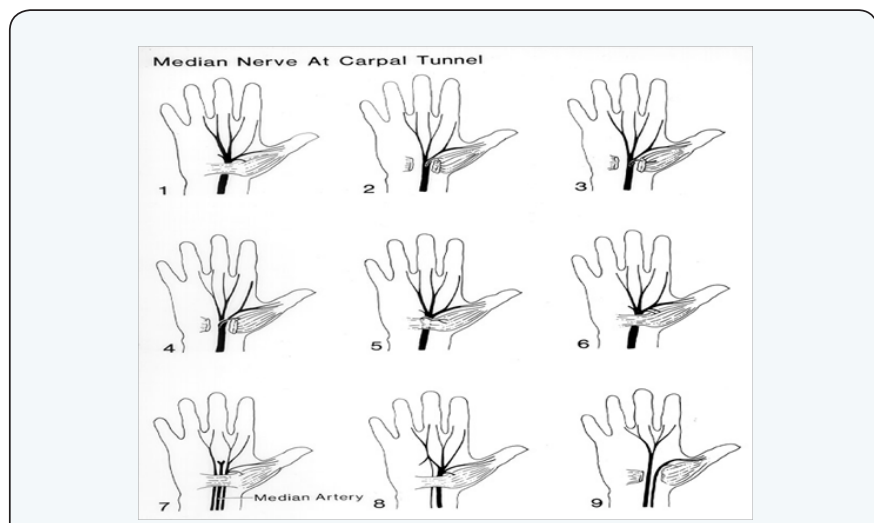

Figure 2:

1: Usual branching of the median nerve.

2: Thenar branch leaving the median nerve within the carpal tunnel (subligamentous).

3: Transligamentous course of thenar branch.

4: Thenar branch leaving median nerve at its ulnar aspect.

5: Thenar branch crosses over the top of the transverse carpal ligament.

6: Doubled thenar motor branch.

7: High division of the median nerve with a persistent median artery between the two parts.

8: High division of the median nerve with a thinner ulnar part.

9: High division of the median nerve with a thicker ulnar part.

Figure 2 represents the variations as described by Poisel and Lanz and the diversity of the path of the median nerve.

Mizia et al. [12] conducted an autopsy study to look at 60 wrists from 30 Cadavers. 43 of the hands from the research had branching of the thenar branch of the median nerve, as previously described and classified by Poisel as being the usual branching of the median nerve or extraligamantous standard group:

i. $\quad 17$ hands had as previously uncategorized variants;

ii. 5 hands had variations with an accessory branch at the distal carpal tunnel;

iii. $\quad 3$ hands showed High division of the median nerve; and

iv. 4 hands showed an accessory branch proximal to the carpal tunnel.

With regards to surgical approaches to the wrist, it would seem evident that the understanding of these variants should be paramount, as - depending upon where the median nerve travels through the transverse ligament -will have direct implications for open carpal tunnel surgery. The surgical practitioner will have a non single standard anatomical model when performing open carpal surgery, and therefore during, dissection will 
encounter a variant form which, upon identification, will guide the course of the surgery [13].

The surgical practitioner should be able to identify anatomical land marks when performing an open carpal tunnel operation. Anatomical landmarks that are useful in open carpal tunnel surgery include Kaplan's line; pisiform; hook of the hamate; and the thenar and hypothenar eminences. Other structures that should be noted are the distal wrist crease; the flexor carpi radialis; and the Palmaris longus. The surgical practitioner should also be aware that when making surgical incisions, the median nerve has small palmer cutaneous branches which should be avoided to prevent palmer sensation loss [14]. Chang suggested that the standard open carpal tunnel operation should be performed by initially making an incision that should be longitudinal or slightly curvilinear and 3-4 cm in length, starting at Kaplan's line and carrying on to the level of the distal wrist crease.

The surgical incision should follow the groove between the thenar and hypothenar eminences and this should be in the central axis of the ring finger. At an angle of 45 degrees to the distal wrist crease, the surgical incision can be extendedproximally $[14,15]$. After the initial surgical incision, the skin, subcutaneous fat, palmar fascia, and the transverse fibres of the flexor retinaculum will be seen. The next identifiable anatomical landmark will be the palmaris longus, which can be retracted radially to expose the next anatomical landmark the flexorretinaculum. By retracting and exposing the Palmaris longus, the palmercutaneous branch of the median nerve can be avoided. The next stage of the procedure is to identify the proximal and distal extent of the flexor retinaculum. The flexor retinaculum's distal border can be identified by its fat pad.

The proximal landmark of the flexor retinaculum can be identified by its ante brachial fascia being very thin in comparison to the thicker, more notable transverse carpal ligament $[14,15]$. The next anatomical landmark will be the median nerve. This will be identified by making an incision before hand of the proximal facia, which will then give rise to visualization of the median nerve. With identification of the median nerve, the flexor retinaculum should be incized longitudinally, proximally too distally. The next anatomical landmark that should be noted is the hook of the hamate. The hook of the hamate is anatomically situated close to the ulnar artery and nerve, and therefore care should be taken upon exploration of these structures [16]. Dorsal to the transverse carpal ligament are the flexor tendons, these should be retracted radially to visualize the carpus. The recurrent motor branch of the median nerve should be identified before closure, and, if an extended radial incision is used, the palmar cutaneous branch of the median nerve should also be identified $[14,15]$.

Cadaveric studies by Johnson and Shrewsbury [17] found that $80 \%$ of dissected hands showed the thenar branch of the median nerve entering a tunnel of its own covered by the transverse ligament. This may suggest in reference to open carpal tunnel procedures that practitioners may be confronted with the thenar branch of median nerve being obscured from view, but with good prior knowledge of variant forms, practitioners may identify the extra tunnel surrounding the thenar branch of the median nerve. The study by Johnson and Shrewsbury [17], however, may have inadvertently called this a variant when potentially it may have been the result of the flexor retinaculum area of the fibrotendinous tissue of the thenar muscle being included in the research results. Senanayake et al. [18] conducted cadaveric research by dissecting 60 hands from both sides of cadavers used in the research. 15 male and 15 female cadaveric specimens where used with an age range from 30-87.

Results from the cadaveric research found that in 53 of the hands, the extraligamantous variant was identified. In 7 hands, the subligamentous variant was identified, and in none of the specimens was the transligamentous variant seen. Senanayake et al. [18] suggested that the transligamentous variant that was not seen in their research but had been previously identified from $7 \%$ to $80 \%$ in other cadaveric research studies such as that by Kozin [19], who identified $7 \%$ of a sample study as having the transligamentous variant; and Lanz [11], who made the observation that the $23 \%$ incidence rate of the variant transligamentous form may be due to miss identification of the distal edge of the flexor retinaculum [20].

Further research by Alizadeh et al. [21] used a cadaveric study looking at 60 hands. Conclusions from the study showed that the median nerve variations could be seen in $78 \%$ of specimens.

i. $\quad 7 \%$ showed the recurrent nerve running along an extraligamantous course;

ii. $\quad 28.3 \%$ showed subligamentous variation;

iii. $\quad 11.7 \%$ showed a transligamentous variant; and

iv. $11.7 \%$ of specimens showed the recurrent branch originating from the ulnar side.

Interestingly, cadaveric research seems to show varied results on the incidence and type of anatomical variants in the travel of the median nerve and its tributaries through the transverse carpal ligament [21]. For the purpose of surgical practice, it would seem essential that surgical practitioners be aware of the variant groups as described and charted by Lanz [11]. Other authors such as Bhandari [22] have examined the actual surgical approach, contrasted open carpal tunnel surgery with laparoscopic, and have suggested that both positives and negatives have been shown in clinical trials from both techniques. However, broader discussion on this matteris beyond the remit of this paper and is merely speculative. It could also be argued that the open carpal approach gives access to the carpal canal 
and structures within, but provides limited access to the mid carpus and the hook of hamate.

The findings emphasize the importance of approaching the median nerve from the ulnar side when opening the carpal tunnel. And that overly the surgical practitioner must be versed in the variant anatomy of the median nerve through the transverse carpal ligament, taking into consideration the complications that could arise from dissection into any of these variants. By looking at various anatomical literatures, it can be seen that a huge statistical diversity has been noted in respect to this area and that it is a heavily researched topic in relation to a procedure that is carried out on a daily basis across the UK and the world. Further study in relation to this work could include comparisons of surgical techniques considering laparoscopic approaches in comparison with open surgery, still considering the types of variant as discussed in this paper and relating it to surgical practice.

\section{References}

1. East Kent Hospitals (2012) Surgical Prognosis.

2. Skandalakis Lee J, Skandalakis John E (2009) Surgical Anatomy and Technique: A Pocket Manual. Springer Publishing, New York, USA.

3. Crouch T (1995) Carpal Tunnel Syndrome and Repetitive Strain Injuries. Berkeley, North Atlantic Books, CA, USA.

4. Luchetti Riccardo, Amadio Peter (2007) Carpal Tunnel Syndrome. Springer Publishing, New York, USA.

5. Snell RS (2012) Clinical Anatomy by Regions. Lippincott Williams and Wilkins, USA.

6. Fraser J Leversedge, Martin I Boyer, Charles A Goldfarb (2009) A Pocket book Manual of Hand and Upper Extremity Anatomy: Primus Manus. Lippincott Williams and Wilkins, USA.

7. Fessler RG, Sekhar L (2006) Atlas of Neurosurgical Techniques: Spine and Peripheral Nerves. Theime Medical Publishing, USA.

8. Oh SJ (2003) Clinical Electromyography: Nerve Conduction Studies. Lippincott Williams and Wilkins, USA.
9. Cooney W (2000) The Wrist: Diagnosis and Operative Treatment. Elsevier Science, USA.

10. Poisel S (1974) Ursprung und Verlauf des Ramus muscularis des Nervus digitalis palmaris communis I (N. medianus) Chir Praxis 18: 471-474.

11. Lanz U (1977) Anatomical variations of the median nerve in the carpal tunnel. J Hand Surg 2(1): 44-53.

12. Mizia E, Klimek-Piotrowska W, Walocha J, Rutowski R, Wojtala R (2011) The median nerve in the carpal tunnel. Folia Morphol (Warsz) 70(1): 41-56.

13. Beasley RW (2003) Surgery of the Hand. Thieme Medical Publishing, New York, USA.

14. Szabo RM (1999) Compression Neuropathies. Operative Hand Surgery ( $4^{\text {th }}$ edn) Churchill Livingstone, New York, USA.

15. Scuderi Giles R, Tria Alfred J (2010) Minimally Invasive Surgery in Orthopaedics. Springer Science, New York, USA.

16. Gordon TA, Cameron JL (2010) Evidence-based Surgery. Springer Publishing, New York, USA.

17. McMinn RMH (2005) Lasts Anatomy Regional and Applied. Oxford: Elsevier Publishing.

18. Senanayake KJ, Salgado S, Fernando R (2009) Course Pattern of the muscular branch of the median in Sri Lankans Hand. Int J Morphol 27(4): 1059-1061.

19. Johnson RK, Shrewsbury MM (1970) Anatomical course of the thenar branch of the median nerve-usually in a separate tunnel through the transverse carpel ligament. J Bone joint Surg Am 52(2): 269-273.

20. Kozin SH (1998) An unusual branch of the median nerve. J Hand Surg Am 23(5): 852-858.

21. Alizadeh K, Lahiji F, Phalsaphy M (2006) Safety of carpal tunnel release with a short incision. A cadaver study. Acta Orthop Belg 72(4): 415-419.

22. Bhandari M (2011) Evidence-based Orthopaedics. Oxford: Wiley Blackwell Chang J (2010) Plastic Surgery: Volume 6: Hand and Upper Limb.USA: Elsevier Science.

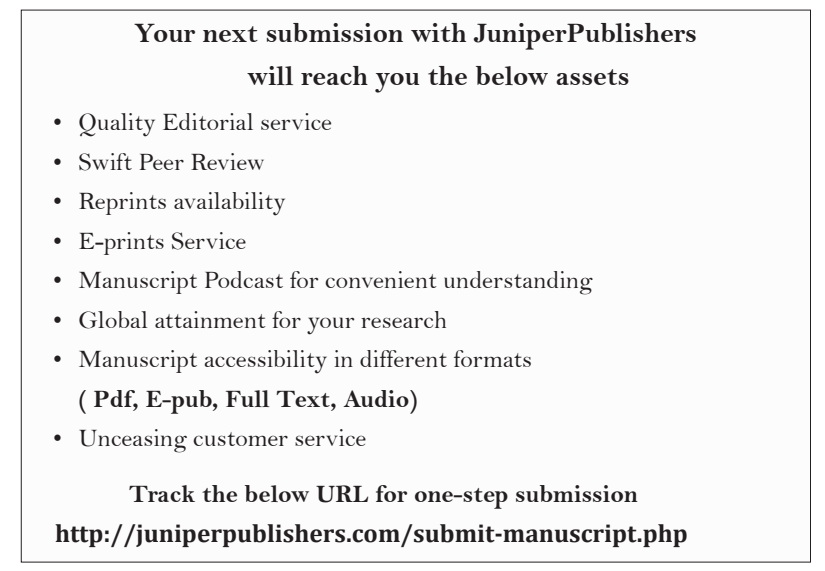

